\title{
Estudio de la Respuesta a los Armónicos de un Sistema Masa Resorte, Caso: Cuasi Resonancia, Resolución
}

\section{Study of the Harmonic Response of a Spring Mass System, Case: Quasi Resonance, Resolution}

DOI: $10.46932 / \mathrm{sfjdv2n5-082}$

Received in: Oct 1st, 2021

Accepted in: Dec 30th, 2021

\author{
J. Agustín Flores Ávila \\ Maestro en Ciencias \\ Instituto Tecnológico de la Laguna \\ Calle Brezo 119 Col. Bellavista \\ Gómez Palacio, Dgo. México. C. P. 35050 \\ E-mail: cidde2010@gmail.com \\ Abel Rodríguez Franco \\ Maestro en Ciencias \\ Instituto Tecnológico de la Laguna \\ Paseo de la Espuela 249, Residencial la Hacienda \\ Torreón, Coah. Méx. C.P. 27276 \\ E-mail: abel.r.f@hotmail.com
}

\section{RESUMEN}

La Cuasiresonancia en los sistemas dinámicos, del tipo Oscilador Mecánico, se presenta cuando la frecuencia del sistema en estudio está "muy próxima" a la frecuencia de la señal de excitación. En estos casos la respuesta presenta la forma de una señal modulada en amplitud (AM) formada por una portadora y un mensaje. Es importante conocer tanto la frecuencia como el período de éstas señales para efecto de tomar previsiones cuando equipo mecánico o construcciones, como puentes o edificios, estén expuestos a esta clase de eventos y así evitar daños cuyas consecuencias tanto en vidas humanas como económicas llegan a ser catastróficas. En este artículo presentamos el procedimiento para obtener el valor de estos parámetros.

Palabras claves: Cuasiresonancia, Oscilador Mecánico, Amplitud Modulada, Portadora, Mensaje, Señal de Excitación.

\begin{abstract}
The Quasi-consonance in dynamical systems, type Mechanical Oscillator, occurs when the frequency of the system under study is "very close" to the frequency of the excitation signal. In these cases the response is in the form of an amplitude modulated (AM) signal consisting of a carrier and a message. It is important to know both the frequency and the period of these signals in order to take forecasts when mechanical equipment or constructions, such as bridges or buildings, are exposed to this type of event and thus avoid damage whose consequences in both human and economic lives become catastrophic. In this article we present the procedure for obtaining the value of these parameters.
\end{abstract}

Keywords: Quasi-resonance, Mechanical Oscillator, Amplitude Modulated, Carrier, Message, Excitation signal. 


\section{ANTECEDENTES}

a) En $\left[{ }^{1}\right]$ p.94 iniciamos el estudio de la respuesta a la frecuencia de un oscilador mecánico haciendo énfasis en la fase de resonancia; por el tipo de señal de excitación este comportamiento fue provocado por el tercer armónico $(n=3)$. La solución obtenida junto con la gráfica la mostramos en la figura 1.

Figura 1.- Función respuesta y gráfica que nos muestra la respuesta de un sistema en estado de resonancia. La resonancia es provocada por el tercer armónico.

$$
\mathrm{x}(\mathrm{t}):=\left[\sum_{n=1}^{2}\left[B(n) \cdot \frac{\mathrm{n} \cdot \sin (3 \mathrm{t})-3 \cdot \sin (\mathrm{n} \cdot \mathrm{t})}{6 \cdot\left(\mathrm{n}^{2}-9\right)}\right]+\left(\frac{\sin (3 \cdot t)}{54}-\frac{\mathrm{t} \cdot \cos (3 \cdot t)}{18}\right)+\sum_{n=4}^{10}\left[B(n) \cdot \frac{n \cdot \sin (3 t)-3 \cdot \sin (n \cdot t)}{6 \cdot\left(n^{2}-9\right)}\right] \cdot \Phi(t)\right.
$$

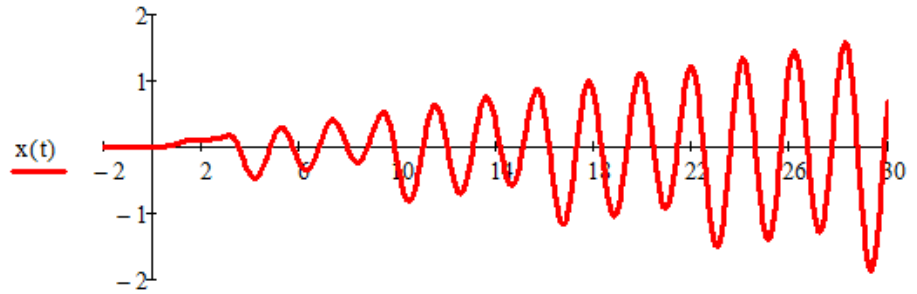

b) En $\left[{ }^{2}\right.$ ] p.2005-2019 continuamos con el estudio de esta clase de sistemas, pero ahora el énfasis lo ponemos en la fase que definimos como "cuasiresonancia"; por el tipo de señal de excitación este comportamiento fue provocado por el primer armónico $(n=1)$. Señalamos el hecho de que el comportamiento anterior, fase de resonancia, es imposible que se presente en la práctica. La solución obtenida junto con la gráfica la mostramos en la figura 2.

Figura 2.- Función y gráfica que nos muestra la respuesta de un sistema en estado de "cuasiresonancia".

$$
x(t):=-\frac{22 \cdot \sin (\pi \cdot t)-2 \cdot \pi \cdot \sqrt{11} \cdot \sin (\sqrt{11} \cdot t)}{11 \cdot \pi^{3}-121 \cdot \pi}
$$

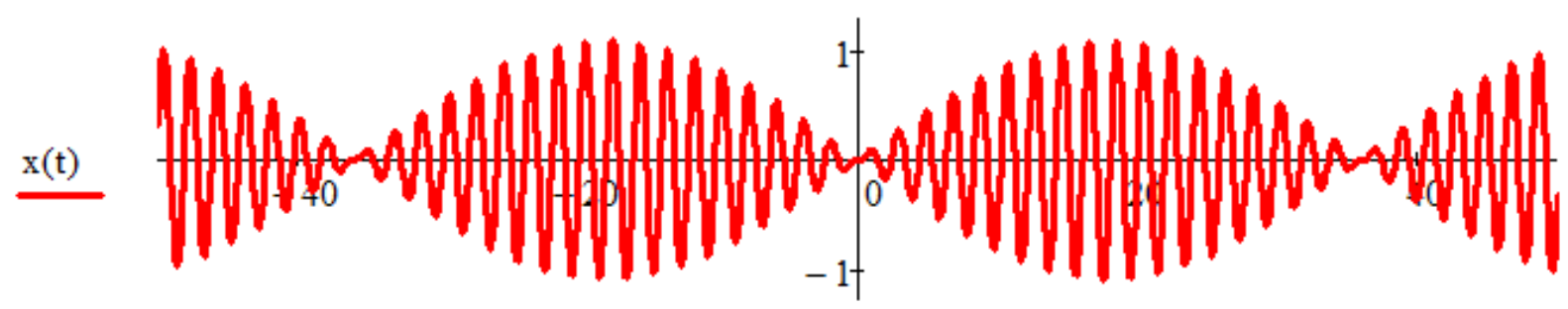


c) En esta entrega hacemos un estudio detallado de la respuesta en estado de cuasiresonancia dando respuesta a una serie de preguntas como: ¿Por qué se da ésta respuesta? . . ¿Cuáles son las frecuencias asociadas? etc.

Recordemos que el análisis lo realizamos a partir de un problema del tipo:

Determine la función $x(t)$ que nos permite conocer la posición en todo instante de la masa m = 1 que está unida a un resorte con " $k=a$ ", si partiendo del reposo y desde el punto de equilibrio, se le aplica una señal de excitación dada por la función:

$\mathrm{f}(\mathrm{t}):=\mid \begin{aligned} & (2-\mathrm{t}) \text { if } 0 \leq \mathrm{t} \leq 2 \\ & \mathrm{f}(\mathrm{t}-\mathrm{T}) \text { if } \mathrm{t}>2\end{aligned}$

Función periódica con período $T=2$ y frecuencia angular $\omega_{0}=\pi$.

Nota: Las unidades se encuentran adecuadamente dimensionadas según el sistema en que se trabaje, sea "cgs o mks".

Y que el problema está diseñado para fines didácticos, es amigable en más de un sentido.

En la resolución empleamos la estrategia señalada en $\left[{ }^{3}\right.$ ] a la luz del escenario que nos presenta la Cuarta Revolución Industrial (Industria 4.0: Science, Technology, Enginnerig \& Mathematics STEM).

\section{RESOLUCIÓN DEL PROBLEMA}

En el estudio señalamos que un sistema opera en estado de "cuasi resonancia" cuando la frecuencia de la señal de excitación tiene un valor "muy próximo" a la frecuencia natural del sistema y es un efecto que se debe evitar, ya que la respuesta es una señal "modulada en amplitud", que para efecto de vibraciones mecánicas implica una oscilación cuya amplitud aumenta dentro de ciertos límites, no siempre dentro del rango de seguridad de los equipos.

En el documento mencionado señalamos que la modulación en amplitud es generada por el primer armónico de la señal $(\mathrm{n}=1)$ y lo mostramos haciendo el estudio correspondiente, obteniendo como función solución general para la posición $\mathrm{x}(\mathrm{t})$ la expresión (1), donde: " $\sqrt{a}$ " es la frecuencia natural del sistema.

$$
\left.x(t, a):=\frac{2}{\pi} \cdot\left[\frac{\sqrt{a} \cdot \sin (\pi \cdot t)-\pi \cdot \sin (\sqrt{a} \cdot t)}{\sqrt{a} \cdot\left(a-\pi^{2}\right)}\right] \ldots 1\right)
$$

Presentamos la solución para el caso en que “a $=11$ ” y obtuvimos la respuesta mostrada en la figura 3 (p. 2015 de $\left.\left[{ }^{2}\right]\right)$. 
Figura 3: Gráfica de la respuesta del sistema en cuasiresonancia: La oscilación va incrementando su amplitud provocando riesgo de destrucción del sistema.

$$
\mathrm{x}(\mathrm{t}, \mathrm{a}):=\frac{2}{\pi} \cdot\left[\frac{\sqrt{11} \cdot \sin (\pi \cdot \mathrm{t})-\pi \cdot \sin (\sqrt{11} \cdot \mathrm{t})}{\sqrt{11} \cdot\left(11-\pi^{2}\right)}\right]
$$

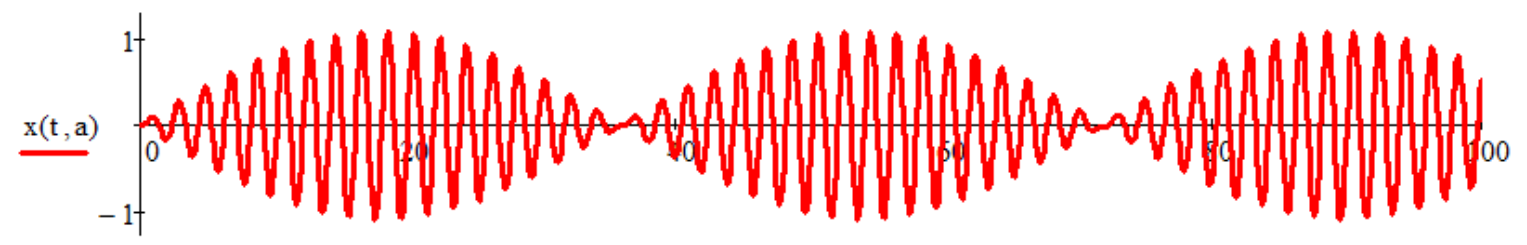

$\mathrm{t}$

\section{OBSERVACIONES}

En la técnica de Amplitud Modulada (AM) en comunicaciones a la frecuencia central se le llama portadora y a la "envolvente" mensaje, por lo que para ser consistentes con esta nomenclatura a la señal interna le llamaremos "portadora" y la envolvente "mensaje" y las identificaremos con el subíndice "p" de portadora y " $m$ " de mensaje; de entrada planteamos dos preguntas:

a).- ¿Cuál es la frecuencia de la portadora y qué la determina?.

b).- ¿Cuál es la frecuencia del mensaje y qué la determina?.

\section{CONJETURA}

El comportamiento cuasiresonante del sistema mecánico, como ya lo señalamos, se origina cuando la frecuencia de la señal de excitación está “mиy próxima” a la frecuencia natural del sistema, sin importar que esté por encima o por debajo; en este problema la frecuencia natural del sistema es " $\sqrt{11}$ " y la frecuencia de la señal de excitación, para el primer armónico, es “ $\pi$ ”; la diferencia entre ellas es: $\sqrt{11}-\pi$ $=0.175$ que es "mиy реquе̃̃a" ([ $\left.{ }^{2}\right]$ página 2014). Planteamos la siguiente conjetura que obtuvimos mediante observación y análisis de la gráfica de la señal solución:

Las frecuencias de la portadora y del mensaje están determinadas por las veces que cabe la diferencia entre los períodos de la señal de excitación y la frecuencia natural del sistema, en cada una de ellas. 


\section{EXPLICACIÓN DE LA CONJETURA}

Esta conjetura la obtuvimos mediante el Método Científico empleado en la resolución de problemas en ciencias. Observamos el evento en estudio, buscamos regularidades, las parametrizamos, las sometimos a validación y repetimos el proceso una y otra vez hasta encontrar la "mejor conjetura"; la validación viene a ser en matemáticas lo que la experimentación es en física.

Imaginémonos a Edison carbonizando su filamento e introduciéndolo en la bobilla una y otra vez hasta que tuvo éxito o a Coulomb observando cómo las bolitas de sauco se atraían o repelían una y otra vez según la magnitud de la carga que lograba añadirles o a Arquímedes sentándose y poniéndose de pie y observando cómo se desplazaba el nivel del agua en la bañera. ¿Qué determinaba que el filamento de Edison iluminara sin arder? ¿Qué determinaba que las bolitas de sauco se atrajeran o repelieran en el caso de Coulomb? ¿Qué determinaba que el nivel del agua en la bañera subiera o bajara según la posición de Arquímedes?

\section{CONSTRUCCIÓN DE LA CONJETURA}

Para el análisis identificamos en la respuesta del sistema dos componentes:

a).- Uno determinado por el primer armónico de la señal de excitación; llamémosle $f_{1}(t)$, y que es (2).

$$
\left.f 1(t):=\frac{2}{\pi} \frac{\sqrt{11} \cdot \sin (\pi \cdot t)}{\sqrt{11} \cdot\left(11-\pi^{2}\right)} \ldots 2\right)
$$

b).- Y otro determinado por la frecuencia natural del sistema; llamémosle $f_{2}(t)$, que es (3)

$$
\mathrm{f} 2(\mathrm{t}):=\frac{-2}{\pi} \cdot \frac{\pi \cdot \sin (\sqrt{11} \cdot \mathrm{t})}{\sqrt{11} \cdot\left(11-\pi^{2}\right)}
$$

Las frecuencias correspondientes son:

$$
\omega_{1}=\pi \quad \text { y } \quad \omega_{2}=\sqrt{11}
$$

Los períodos correspondientes son:

$$
\mathrm{T}_{1}=2 \quad \mathrm{y} \quad \mathrm{T}_{2}=\frac{2 \pi}{\sqrt{11}}
$$


En esta parte recurrimos al método de resolución empleado en los problemas que se estudian en el Nivel Medio Superior sobre el mínimo común múltiplo $(\mathrm{mcm})$ de dos cantidades, del tipo:

\section{PROBLEMA}

Se tienen dos campanas " $A$ " $y$ " $B$ " que tocan de manera simultánea a las 7.00 A. M.; si la campana " $A$ " repica cada 25 minutos y la " $B$ " lo hace cada 40 minutos: ¿Después de cuánto tiempo volverán a coincidir en su repique?

En nuestro problema tenemos dos funciones $\mathrm{f}_{1}(\mathrm{t})$ y $\mathrm{f}_{2}(\mathrm{t})$ que en $\mathrm{t}=0$ se encuentran en $\mathrm{x}=0$; la primera regresa a $\mathrm{x}=0$ después de 2 segundos y la segunda lo hace después de $2 \pi / \sqrt{11}$ segundos (Fig. 4): pregunta ¿Cuánto tiempo tardaran en coincidir en $\mathrm{x}=0$ por primera vez después del inicio ?. En la respuesta a esta pregunta se hace presente el concepto del $\mathrm{mcm}$.

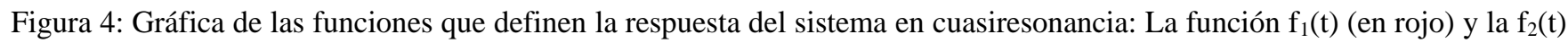
(en azul) coinciden en el punto $(0,0)$ : La roja después de 2 segundos regresa a $\mathrm{x}=0$ y la azul lo hace después de $2 \pi / \sqrt{11}$ segundos: ¿Cuál es el tiempo $\mathrm{t}_{1}$ en el que coinciden por primera vez en el punto $\mathrm{x}=0 ;\left(\mathrm{t}_{1}, 0\right)$ ?

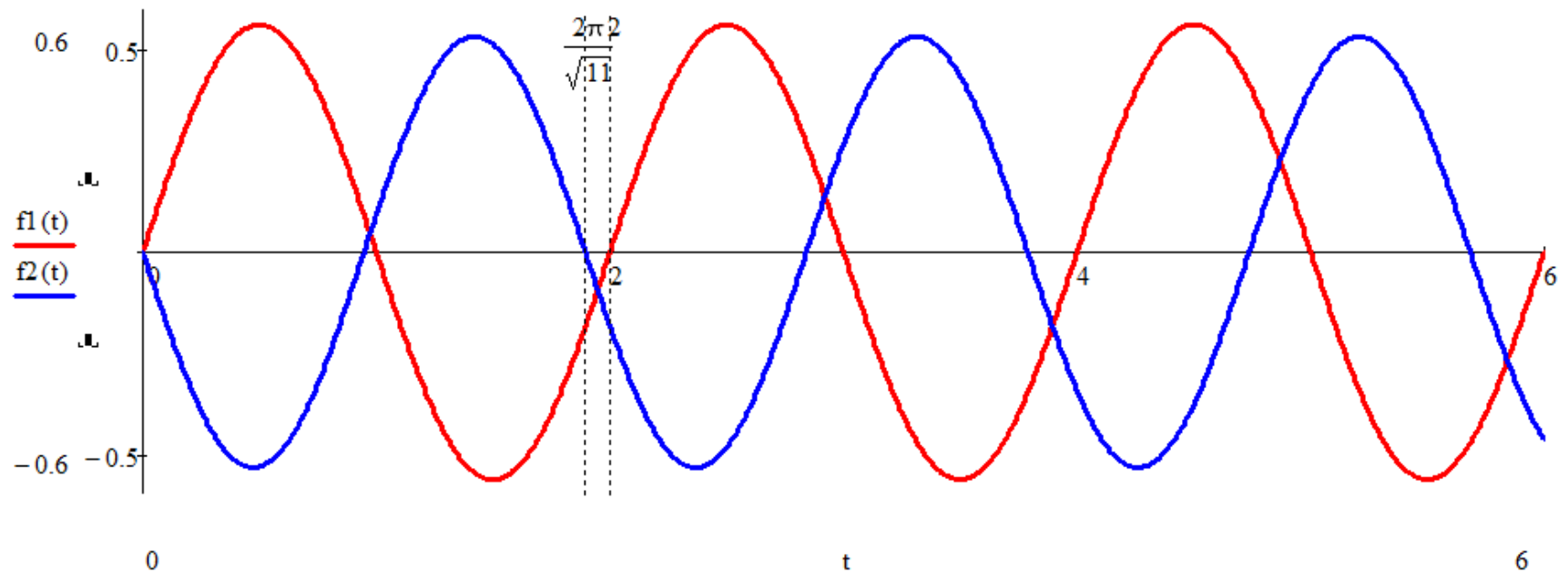

Nuestra conjetura se basa en suponer que la pequeña diferencia entre las señales se presenta como una fuerza adicional que durante los ciclos positivos empuja a la masa alcanzando un máximo y durante los ciclos negativos la va deteniendo, regresándola a cero, determinando así el mensaje de la señal respuesta.

\section{VALIDACIÓN DE LA CONJETURA}

Continuando con lo señalado líneas arriba, en la respuesta del sistema tenemos dos componentes:

$$
\mathrm{f1}(\mathrm{t}):=\frac{2}{\pi} \frac{\sqrt{11} \cdot \sin (\pi \cdot \mathrm{t})}{\sqrt{11} \cdot\left(11-\pi^{2}\right)} \quad \mathrm{f} 2(\mathrm{t}):=\frac{-2}{\pi} \cdot \frac{\pi \cdot \sin (\sqrt{11} \cdot \mathrm{t})}{\sqrt{11} \cdot\left(11-\pi^{2}\right)}
$$


Con sus frecuencias y períodos correspondientes:

Frecuencias:

$$
\omega_{1}=\pi \quad \text { y } \quad \omega_{2}=\sqrt{11}
$$

Períodos:

$$
\mathrm{T}_{1}=2
$$

$$
\mathrm{T}_{2}=\frac{2 \pi}{\sqrt{11}}
$$

La diferencia entre períodos es:

$$
\mathrm{T}_{1}-\mathrm{T}_{2}=2-\frac{2 \pi}{\sqrt{11}}=\frac{2 \cdot \sqrt{11}-2 \pi}{\sqrt{11}} ; \quad \frac{2 \cdot \sqrt{11}-2 \pi}{\sqrt{11}}=0.10554835
$$

Calculamos el número de veces que cabe la diferencia de períodos en el período de la frecuencia natural del sistema; a esta magnitud le llamamos "c"; empleando el método de las conjeturas y el método de prueba y error, encontramos que el doble de esta magnitud "c" es el tiempo en el que se empalman por primera vez las dos señales y determina el período del mensaje $\left[\mathrm{T}_{\mathrm{m}}\right]$ y por lo tanto su frecuencia $\left[\mathrm{f}_{\mathrm{m}}\right]$.

$$
\frac{\frac{2 \pi}{\sqrt{11}}}{\frac{2 \cdot \sqrt{11}-2 \pi}{\sqrt{11}}} \text { simplify } \rightarrow \frac{\pi}{\sqrt{11}-\pi}
$$

Valor "aproximado"

$$
\frac{\pi}{\sqrt{11}-\pi}=17.948662
$$

El doble es el período del mensaje y el inverso es la frecuencia, es decir, la frecuencia natural del sistema determina la frecuencia del mensaje:

$$
\mathrm{T}_{\mathrm{m}}=2 \cdot\left(\frac{\pi}{\sqrt{11}-\pi}\right) \quad \mathrm{T}_{\mathrm{m}}=35.8973239
$$




$$
\mathrm{f}_{\mathrm{m}}=\frac{\sqrt{11}-\pi}{2 \pi}
$$

$$
f_{m}=0.0278572
$$

Este resultado lo mostramos en las figuras 5 y 6.

$$
\mathrm{x}(\mathrm{t}):=\frac{2}{\pi} \cdot\left[\frac{\sqrt{11} \cdot \sin (\pi \cdot \mathrm{t})-\pi \cdot \sin (\sqrt{11} \cdot \mathrm{t})}{\sqrt{11} \cdot\left(11-\pi^{2}\right)}\right] \quad \mathrm{c}:=\left(\frac{\pi}{\sqrt{11}-\pi}\right)
$$

Figura 5: Gráfica de la respuesta del sistema en cuasiresonancia: Cuantificamos el período de la envolvente de la respuesta y por lo tanto su frecuencia. En el tiempo "2c" se completa un ciclo de la envolvente.

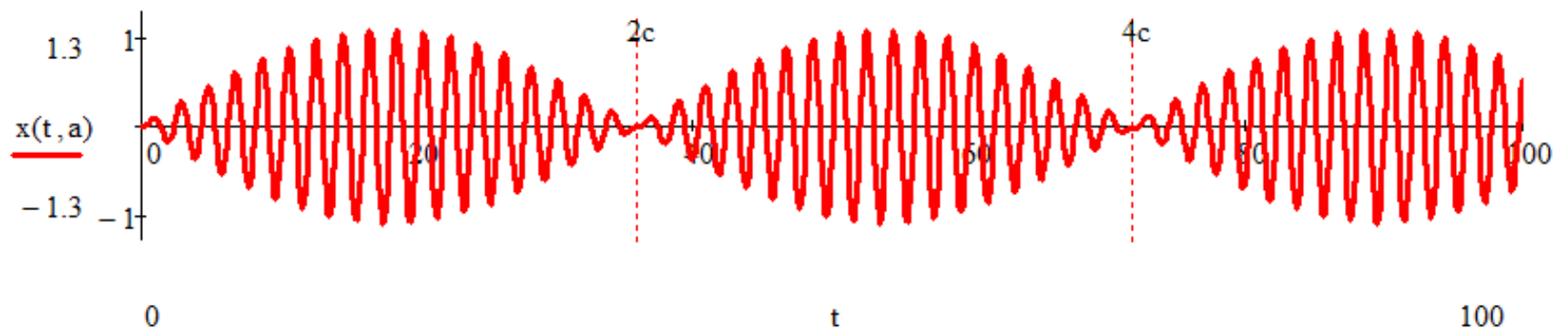

Figura 6: Gráfica de la respuesta del sistema en cuasiresonancia: Ampliamos el rango del tiempo y verificamos que efectivamente se satisface la conjetura. Cada " $2 c$ " se repite la envolvente. Por supuesto que es una evidencia empírica, pero: ¿Cuándo, sino es en estas ocasiones en las que las TIC's son de gran ayuda y apoyo en la resolución y estudio de cierta clase de problemas?.

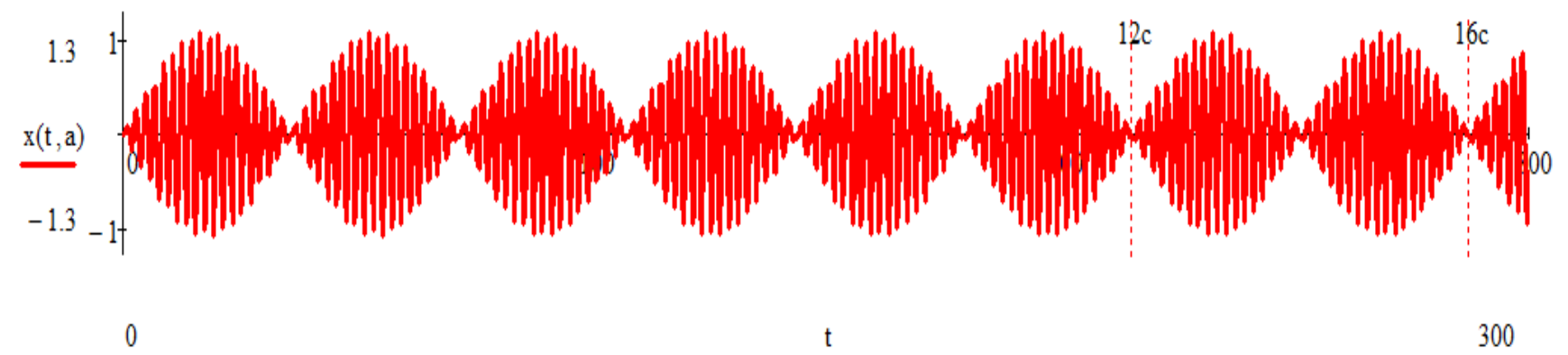

Ahora calculamos el número de veces que cabe la diferencia de períodos en el período del primer armónico; nuevamente haciendo conjeturas y mediante prueba y error encontramos que este valor determina el número de ciclos que caben en el mensaje.

$$
\frac{2}{\frac{2 \sqrt{11}-2 \pi}{\sqrt{11}}} \text { simplify } \rightarrow \frac{\sqrt{11}}{\sqrt{11}-\pi} \quad ; \quad \frac{\sqrt{11}}{\sqrt{11}-\pi}=18.948662 \text { । }
$$


Este es el número de veces que cabe la diferencia de períodos en un ciclo del mensaje y es igual al número de ciclos de la portadora que caben ahí.

Ahora, para calcular la frecuencia y el período de la portadora consideramos lo siguiente: Si en un período de tiempo "A" caben "B" ciclos de una señal, entonces, el cociente $\mathrm{A} / \mathrm{B}$ nos proporciona la duración de cada ciclo, es decir, su período, por ejemplo, si en 20 segundos caben 5 ciclos, cada ciclo dura 4 segundos y ese es su período.

Por lo tanto, si el período del mensaje es:

$$
\mathrm{T}_{\mathrm{m}}=2 \cdot\left(\frac{\pi}{\sqrt{11}-\pi}\right)
$$

Y el número de veces que cabe la diferencia de períodos en el mensaje es:

$$
\frac{\sqrt{11}}{\sqrt{11}-\pi}
$$

Los cocientes de estas dos magnitudes nos proporcionan el período y la frecuencia de la portadora que, como podemos observar, es igual a la frecuencia natural del sistema:

$$
\begin{gathered}
\frac{2 \pi}{\frac{\sqrt{11}-\pi}{\sqrt{11}}}=\frac{2 \pi}{\sqrt{11}} \\
\mathrm{~T}_{\mathrm{p}}=\frac{2 \pi}{\sqrt{11}-\pi}=1.8944517
\end{gathered}
$$

El inverso es la frecuencia de la portadora.

$$
f_{p}=\frac{\sqrt{11}}{2 \pi}=0.5278572
$$

Este resultado lo mostramos en la figura 7. 
Figura 7: Gráfica de la respuesta del sistema en cuasiresonancia: Si observamos la gráfica y contamos el número de ciclos dentro de un ciclo del mensaje, efectivamente el número anda por ahí de los 18.

$$
\mathrm{x}(\mathrm{t}, \mathrm{a}):=\frac{2}{\pi} \cdot\left[\frac{\sqrt{11} \cdot \sin (\pi \cdot \mathrm{t})-\pi \cdot \sin (\sqrt{11} \cdot \mathrm{t})}{\sqrt{11} \cdot\left(11-\pi^{2}\right)}\right] \quad \frac{\sqrt{11}}{\sqrt{11}-\pi}=18.948662
$$

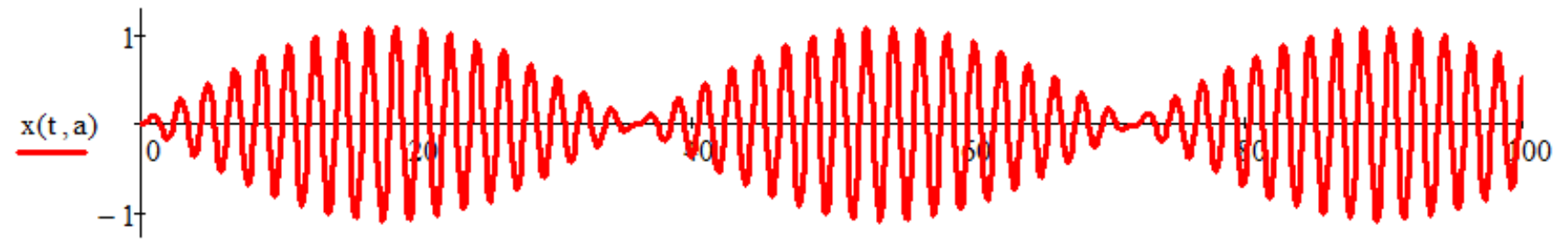

$\mathrm{t}$

\section{OBSERVACIÓN}

Este mismo problema lo resolvimos para valores diferentes de "a" (con $\sqrt{a}$ frecuencia natural del sistema), por encima y por debajo del primer armónico “ $\pi$ ” y los resultados son consistentes con la conjetura.

El escenario sería un conjunto de estructuras como, edificios, torres, puentes, etc. que se ven sometidos a una fuerza externa, producto de, por ejemplo, un temblor; cada una de ellas experimentará una afectación diferente dependiendo de su frecuencia natural. Es una misma fuerza caracterizada por su frecuencia actuando sobre sistemas con diferente frecuencia natural por lo que las respuestas serán diferentes: Esta quedó incólume; aquella sufrió daños menores pero la de más allá se derrumbó, figura 8.

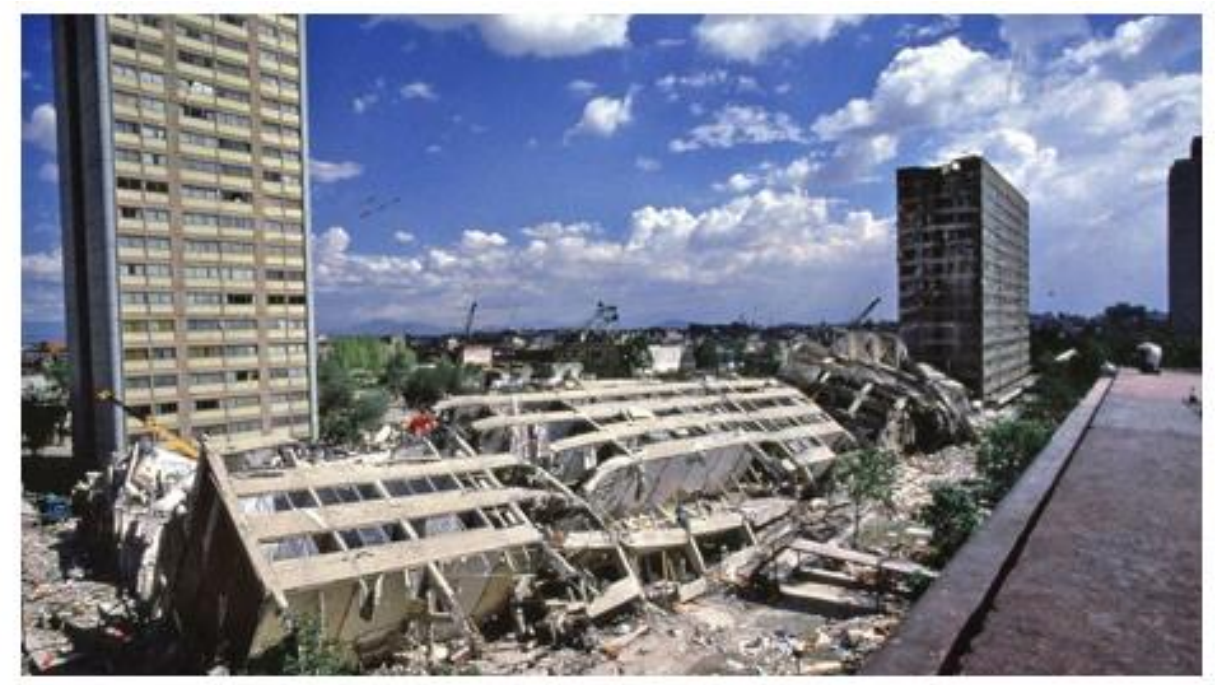

Figura 8: Foto en la que se muestran los años diferenciados que deja un terremoto en los alrededores dependiendo del tipo de construcción. El edificio Nuevo León se destruyó por completo y el Veracruz no sufrió daños. ¿Frecuencias naturales diferentes 0 


\section{CONCLUSIÓN}

Proponemos que los múltiples casos de destrucción de sistemas como [4, 5, 6 y 7] que se tienen registrados, por supuesto efecto de la resonancia, son más bien efecto de la cuasiresonancia en los que la vibración, desplazamiento de los cuerpos, llega a un punto en el que el cuerpo/sistema se destruye. 


\section{REFERENCIAS}

1. $\quad$ https://www.smm.org.mx/files/2018/programa_extenso_2018.pdf

2. https://southfloridapublishing.com/ojs/index.php/jdev/issue/view/13

3. https://www.youtube.com/watch?v=xMMdnA8AV9Y

4. https://www.youtube.com/watch?v=sH7XSX10QkM\&feature=youtu.be

5. https://www.youtube.com/watch?v=Oc27GxSD bI

6. https://www.youtube.com/watch?v=ior-xQMENpc

7. $\quad$ https://www.youtube.com/watch?v=ior-xQMENpc

8. Nota: Para bibliografía y referencias complementarias, consúltense 1, 2 y 3. 